\title{
Augmented Lagrangian procedure for implicit computation of contact-impact between deformable bodies.
}

\author{
D Graillet, J-P Ponthot, L Stainier \\ LTAS - Milieux Continus et Thermomécanique, Université de Liège, Chemin des Chevreuils 1, 4000 Liège, Belgium
}

\begin{abstract}
This paper shows how efficiency can be improved by using an adequate Augmented Lagrangian procedure instead of the classical and well-known Penalty method for solving contact-impact problems between deformable bodies, including frictional contact, large deformations, dynamical effects and inelasticity phenomena. The Augmented Lagrangian method has already enjoyed great success in solving constrained minimisation problems or incompressibility conditions. Alternatives to existing automation techniques for augmentations are presented. Starting from a Penalty method, it will be seen how the Augmented Lagrangian decreases ill-conditioning of governing equations and gives a more precise solution with a lower CPU-cost. Several original simultaneous criteria are proposed for optimising the number and the location of the augmentations in an incremental implicit resolution. Application of the method is done for two axisymmetric impact problems.
\end{abstract}

\section{NOTATION}

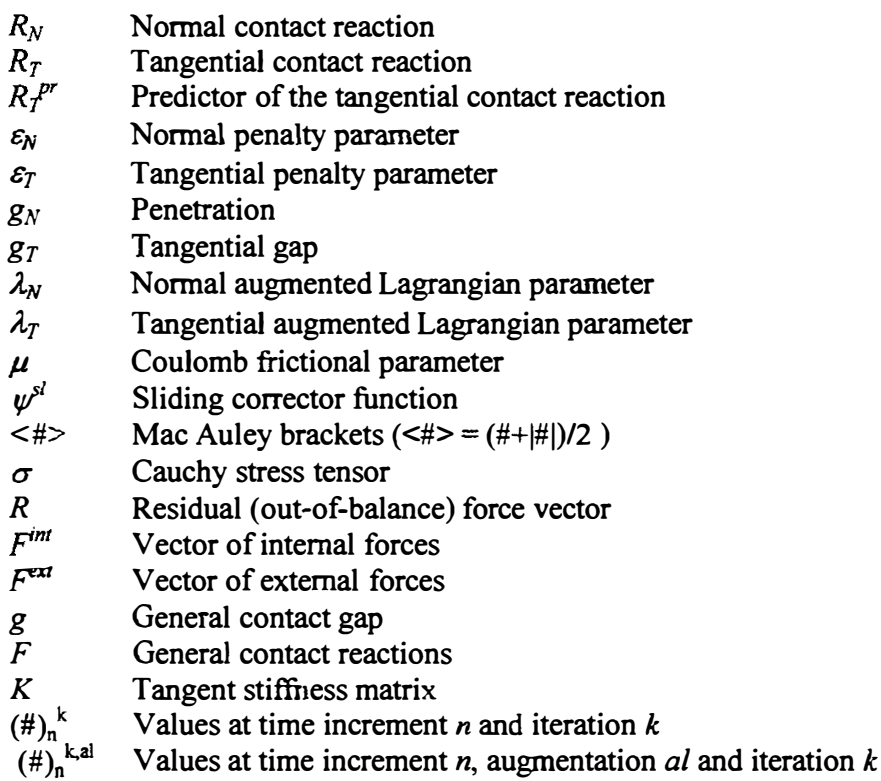

\section{INTRODUCTION}

This work is concerned with the large deformation processes including mechanical and frictional contact. These can be encountered for instance in metal forming, crashworthiness or tribology. The constraints linked to this contact problem are impenetrability, expressed as a set of complementarity conditions, and friction. One usually chooses an analytical method to solve this constrained problem (Lagrange multiplier method), or the Penalty regularisation method that doesn't need any approximation in the stick-slip transition or simplification in loading-unloading conditions. The problem is the high sensitivity of the solution and the CPU-cost with respect to the penalty parameters: higher penalties give better solutions while risk of ill-conditioning rapidly degrades the convergence. In response to this drawback, some authors ([8-11]) proposed the Augmented Lagrangian method that can be seen like an adequate compromise between the Lagrange multipliers method and the Penalty method, possessing the best characteristics of Penalty and Lagrange multipliers algorithms. One of the open questions in augmented Lagrangian formulation is how to automatically stop the augmentation process. In this paper we present an original criterion which aims at stopping automatically the augmentation process while keeping this number as low as possible. A thorough comparison of the precision of both Penalty and Augmented Lagrangian algorithms is also presented. 


\section{FROM PENALTY TO AUGMENTED LAGRANGIAN}

\section{The Penalty method}

In the Penalty method, the nodal reactions, resulting from contact between a contact node and a contact surface, are directly proportional to the penetration (orthogonal component) and, in the case of sticking contact, to the tangential displacement (tangential component) along the surface encountered. Since this method is a regularisation method based on the penalty principle, it does not exactly fulfil the conditions of impenetrability and perfect sticking: the violation of these two conditions is therefore penalised through the penalty coefficient.

In fact, the penalty method needs some non zero penetration to generate a normal contact force (one can physically describe this penetration in terms of an elastic deformation of the contact surface), as well as some tangential motion to generate the tangential forces, even if the contact is sticking (here again, this might correspond to some elastic shear deformation of the asperities produced by the tangential forces). From a practical point of view, the penalty parameters have to be large enough to reduce these violations to a very small proportion of the total deformation. This means that the contact will not involve penetrations which are too large or sticking point that are too far from the point where they should be to exactly fulfil the non-sliding conditions.

The choice of the penalty parameters strongly influences the precision of the modelisation of the contact situation: the larger the penalties and the smaller the gaps. The best choice would then be to use very large penalty parameters to best fit the impenetrability condition. The problem is that higher penalty parameters create a crescent ill-conditioning of the stiffness matrix that makes the process more demanding with respect to time steps and iterations to solve the problem (with a better accuracy though).

\section{The Penalty equations}

The Penalty method yields for Coulomb friction:

$$
\begin{aligned}
& R_{N}=\left\langle-\varepsilon_{N} g_{N}\right\rangle \\
& R_{T}^{p r}=-\varepsilon_{T} g_{T} \\
& \psi^{s l}=\mu\left\|R_{N}\right\|-\left\|R_{T}^{p r}\right\| \\
& R_{T}=R_{T}^{p r}-\left\langle-\psi^{s l}\right\rangle \operatorname{sign}\left(R_{T}^{p r}\right)
\end{aligned}
$$

The normal contact reaction $R_{N}$ and the predictor of the tangential contact reaction $R_{T}^{p r}$ are simply proportional to their respective gap $g_{N}$ and $g_{T}$. These two quantities are first evaluated. At this stage, the contact is assumed to be sticking. Then the norm of $R_{T}^{p r}$ is inserted into the Coulomb criterion. If the frictional force is smaller than the friction coefficient $\mu$ times the normal force, then the contact is sticking and nothing else is done. On the contrary, if Coulomb's criterion is violated, the final state of the contact is sliding and the predictor of the tangential forces has to be corrected in order to restore consistency. This is done thanks to the sliding corrector $\psi^{s l}$ according to relations (lc \& d). This predictor/corrector algorithm thus ensures a correct and smooth transition between sticking and sliding contact. More details about this algorithm, which is analogous to the elastic predictor/plastic corrector algorithm of elasto-plasticity, generally known as the radial return algorithm, can be found in [9]. 


\section{The Penalty algorithm}

One can write the Penalty algorithm in the following way:

1. Loop on the increments (n) ;

Update of the loading;

2. Equilibrium loop (k) up to $k=\max _{k}$;

Computation of stresses and internal forces:

$$
\sigma_{n}^{k}, R_{n}^{k}=F^{e x t}-F^{i n t} ;
$$

Computation of the contact gap and reactions:

$$
g_{n}^{k}, F_{n}^{k}
$$

Check for equilibrium:

$$
\text { ? } R_{n}^{k}+F_{n}^{k}=0 \text { ?; }
$$

If equilibrium convergence go to 3 ;

Computation of the tangent stiffness matrix:

$$
K_{n}^{k}
$$

Solution using Newton-Raphson method of

$$
K_{n}^{k} d u^{k+1}=R_{n}^{k}+F_{n}^{k}
$$

Update of $\Delta u_{n}^{k+1}=\Delta u_{n}^{k}+d u^{k+1}$;

$k=k+1$;

go to 2

3. Check to stop the computation ;

If stop go to END

$n=n+1, k=0$;

END ;

go to 1 ;

\section{The Augmented Lagrangian method}

One of the commonly accepted drawbacks of the Penalty formulation is that the results often depend on the choice of the penalty parameters. In many situations, this optimal parameter is not obvious to choose. In order to circumvent the penalty sensitivity issue, some alternative formulations, such as the Lagrange multiplier methods are available. The regularisation method considered in this work is the Augmented Lagrangian method. This method can easily be compared to the Penalty formulation with a simple glance at the following equations.

The Augmented Lagrangian equations

$$
\begin{aligned}
& R_{N}=\left\langle\lambda_{N}-\varepsilon_{N} g_{N}\right\rangle \\
& R_{T}^{p r}=\lambda_{T}-\varepsilon_{T} g_{T} \\
& \psi^{s l}=\mu\left\|R_{N}\right\|-\left\|R_{T}^{p r}\right\| \\
& R_{T}=R_{T}^{p r}-\left\langle-\psi^{s l}\right\rangle \operatorname{sign}\left(R_{T}^{p r}\right)
\end{aligned}
$$

The terms $\lambda_{N}$ and $\lambda_{T}$ are called the Augmented Lagrangian multipliers. They're not additional unknowns, as in traditional Lagrangian multiplier methods, which have to be determined simultaneously with displacement degree of freedom, thereby increasing the solution costs. Instead, they are iteratively set to the approximation of the surface tractions obtained during the convergence process. Thus, a different method for finding the multipliers has to be introduced. The key idea in the 
solution scheme is to replace the evaluation of contact forces $\lambda_{N}$ and $\lambda_{T}$ by an iterative update formula, which renders the multipliers known constant quantities during each equilibrium iteration of the process. The series of $\lambda_{N}$ and $\lambda_{T}$ thus converges to the exact solution (zero penetration as far as the normal gap is concerned) for finite values of the penalty parameters. This can be accomplished by nesting the equilibrium loop of the penalty algorithm (point 2 of the algorithm presented above) into what is called the augmented Lagrangian loop (see point 2 of the algorithm hereafter). Therefore, the value of the multipliers is only modified during the augmentation procedure and is kept constant during each equilibrium loop. One of the major advantages of this procedure is that it leaves unaffected the global tangent stiffness matrix of the system. Thus, successive augmentations very easily generate a series of updated values for the multipliers. This series can be shown, for finite penalty parameters, to converge to the value that would be found (both for forces as well as geometrical contact constraints) by a classical Lagrange Multiplier Methodology [12]. The main advantage of the algorithm however is that you don't have to deal with additional unknowns that introduce numerical difficulties associated to the handling of zero terms created on the diagonal of the system matrix. As far as friction is concerned, the sliding correction is identical to the previous correction done for the Penalty method. Thus, at each augmentation, we have:

$$
\left(\lambda_{n}^{a l+1}\right)_{N, T}=\left(F_{n}^{k, a l}\right)_{N, T}
$$

so that the multipliers are made equal to the current contact forces computed in the equilibrium iteration loop thanks to the penalty algorithm. This updating, in turn, introduces a perturbation in the equilibrium equations. After the augmentation, during the next equilibrium loop (still in the same time step), this perturbation will force the contact gap to vanish progressively to zero.

\section{The Augmented Lagrangian algorithm}

One can write the Augmented Lagrangian algorithm in the following way:

1. Loop on the increments (n) ;

Update of the loading ;

2. Augmented Lagrangian loop (al) up to $a l=\max _{a l}$;

3. Equilibrium loop (k) up to $k=\max _{k}$;

Computation of stresses and internal forces:

$$
\sigma_{n}^{k, a l}, R_{n}^{k, a l}=F^{e x t}-F^{i n t} ;
$$

Computation of the contact gap and reactions:

$$
g_{n}^{k, a l}, F_{n}^{k, a l} ;
$$

Check for equilibrium:

$$
? R_{n}^{k, a l}+F_{n}^{k, a l}=0 ? ;
$$

If equilibrium convergence go to 4 ;

Computation of the tangent stiffness matrix:

$$
K_{n}^{k, a l} \text {; }
$$

Solution using Newton-Raphson method of

$$
\begin{aligned}
& \qquad K_{n}^{k, a l} d u^{k+1, a l}=R_{n}^{k, a l}+F_{n}^{k, a l} \\
& \text { Update of } \Delta u_{n}^{k+1, a l}=\Delta u_{n}^{k, a l}+d u^{k+1, a l} ; \\
& k=k+1 ; \\
& \text { go to 3; }
\end{aligned}
$$

4. Check to stop the augmentations ;

If stop go to 5 ;

Update the Augmented Lagrangian coefficients ; 


$$
\begin{aligned}
& \qquad\left(\lambda_{n}^{a l+1}\right)_{N, T}=\left(F_{n}^{k, a l}\right)_{N, T} \\
& \text { al =al+1,k=0;} \\
& \text { go to 3 } ;
\end{aligned}
$$

5. Check to stop the computation ;

If stop go to END

END ;

$n=n+1, a l=0, k=0$;

go to 1 ;

\section{Check to stop the augmentations}

In a given Augmented Lagrangian loop, the augmentation process, see relation (3), is repeated until all contact constraints are satisfied within a tolerance, or little change in the solution vector from augmentation to augmentation is noted. The simplest way to do this is to a priori define a fixed number of augmentation. Laursen and Oancea [7] proposed a more sophisticated procedure for automation of the augmentations based on some mean value of the variation of the Augmented Lagrangian coefficients or a mean value of a relative geometric defect (penetration / sliding) for all the contact nodes. One of the drawbacks of this approach is that, when many nodes are already in contact, and when a new node comes into contact, the local multipliers associated to that node are not efficiently taken into account into the global criteria. To overcome that drawback, we propose the original following criterion based on local values.

In the present formulation, in a given time step, the augmentation process is stopped when the following three conditions are met:

1. Maximal geometrical error allowed (penetration and sticking gap):

$$
\begin{array}{ll}
\max \left(\left|g_{N}\right|\right)<g^{\max } & \text { for sliding contact or } \\
\max \left(\sqrt{g_{N}^{2}+g_{T}^{2}}\right)<g^{\max } & \text { for sticking one }
\end{array}
$$

2. Minimal relative variation of the Lagrangian coefficients necessary:

$$
\begin{aligned}
& \max \left(\frac{\left|\Delta \lambda_{N}\right|}{\lambda_{N}}\right)<\Delta^{\min } \quad \text { for sliding contact or } \\
& \max \left(\sqrt{\left(\frac{\left|\Delta \lambda_{N}\right|}{\lambda_{N}}\right)^{2}+\left(\frac{\left|\Delta \lambda_{T}\right|}{\left|\lambda_{T}\right|}\right)^{2}}\right)<\Delta^{\min } \text { for sticking one }
\end{aligned}
$$

The default value for $\Delta^{\mathrm{min}}$ is set to $10^{-6}$.

3. Maximal number of augmentation allowed:

$$
a l>a l^{\max }
$$

The default value is set to 10 .

The first criterion is an image of the desired geometrical quality of the solution, the second one circumvents useless augmentations (not enough variation of the Lagrangians multipliers to improve significantly the solution) and the third one is an image of the price accepted to be paid for solving the problem (allowing more augmentations can lead to a more expensive but more accurate solution). Practically, at each time step, after an equilibrium configuration has been reached, one more augmentation is performed until one of the above three criteria is violated. The, the solution proceeds to the next time step. 


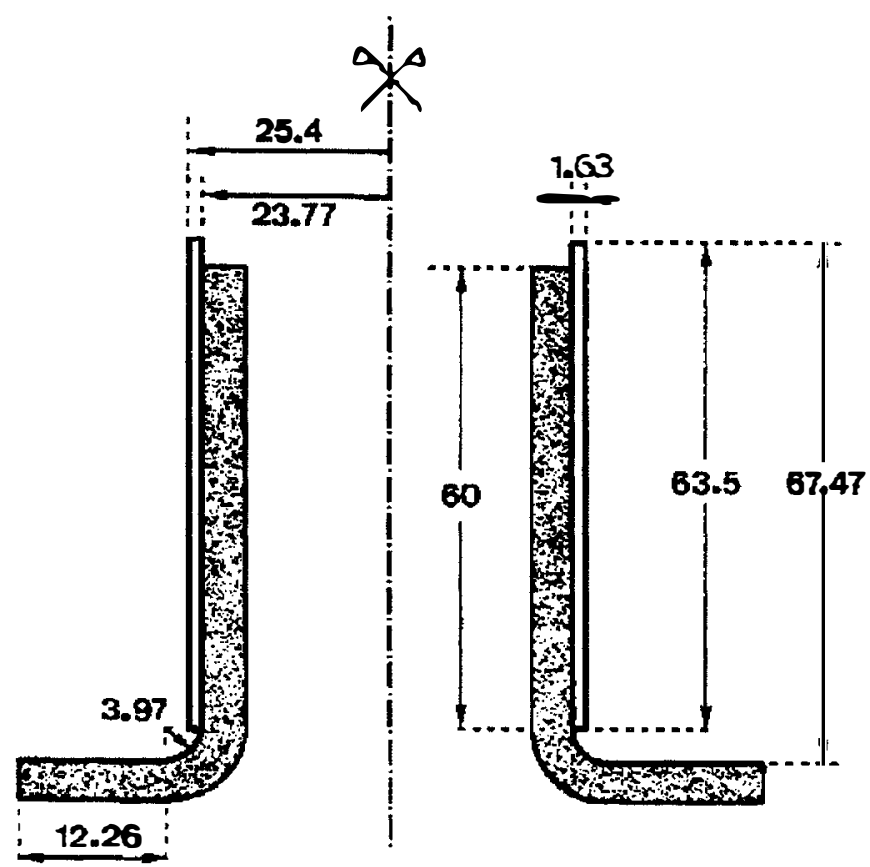

Figure 1: Axisymmetric shock absorber device. Initial geometry. All dimensions are in $\mathrm{mm}$. The shaded area is considered to be rigid

\section{NUMERICAL EXAMPLES}

\section{Introduction}

As a numerical illustration of these concepts, the behaviour of two types of shock-absorber devices has been investigated. The finite element package METAFOR [3] was used. This finite element package is able to simulate quasi-static and transient large deformation problems with complex material behaviour, as well as frictional contact.

Let us point out that during our numerical experiments, time steps size for implicit schemes has not been guided by physical concepts related to frequency contents of the response but mainly by convergence purposes linked with the limited radius of convergence of the Newton-Raphson algorithm.

\section{Shock absorber device}

This first example deals with the numerical modelling of a shock absorber device. It is based on the turning inside-out of a thin walled ductile metal tube. This is generally called an "invertube" device. In this case, a plain tube is confronted with a hard curved die to produce the inversion, figure 1.

This inversion, in turn, produces very large plastic strains which form an efficient energy absorbing mechanism during impact. In this way, the kinetic energy of the impacting bodies is dissipated through plastic deformation, in a controlled fashion at an acceptable rate. The yield limit of the material keeps the transmitted force below an acceptable upper-bound. Hence, the deceleration is slower and less harmful for the people inside the car.

Numerical modelling of the collapse of such energy dissipating structures requires not only to take into account the plastic behaviour of the tube material, as well as inertial forces, but also to consider very large strains and large amplitude rigid body motions that develop and also, in this case, the accurate prediction of frictional forces. Thus a great number of advanced code capabilities are tested by running this kind of problems. 

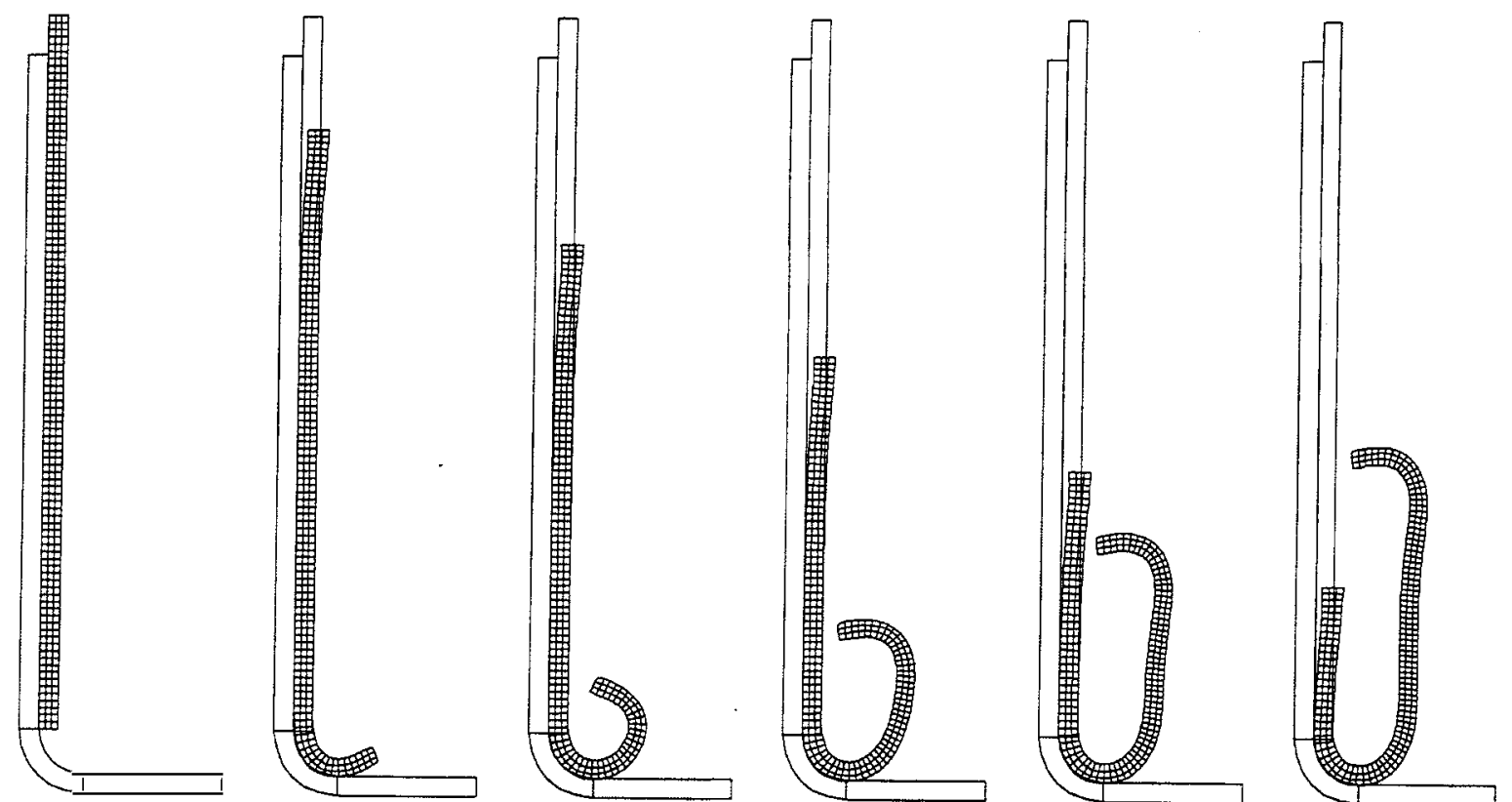

Figure 2: Deformed configurations (frictionless case) for $\mathrm{t}=0.00, \mathrm{t}=0.25, \mathrm{t}=0.5, \mathrm{t}=0.75, \mathrm{t}=1.00$ and $\mathrm{t}=1.25$ milliseconds

Similar problems were investigated by Beltran and Goicolea [4], by Garcia-Garino [5] with an explicit scheme and by Ponthot \& Hogge [2] who compared the performances of explicit and implicit algorithms for frictionless impact problems. More recently, Graillet \& Ponthot [8] made a comparison of several implicit integration schemes versus the explicit one for this frictional problem.

The material consists of an aluminium tube of $50.8 \mathrm{~mm}$ outside diameter times $63.5 \mathrm{~mm}$ length times $1.63 \mathrm{~mm}$ wall thickness. The material is supposed to behave like a J2 elastic-plastic material with linear isotropic hardening. The material parameters are given in table 1 and numerical parameters for the time marching algorithms are given in table 2 . For this simulation, the tangential penalty parameters is always taken as the tenth part of the normal one: $\varepsilon_{T}=\varepsilon_{N} / 10$, with this normal penalty varying from $\varepsilon_{N}=10^{7}$ to $10^{10} \mathrm{~N} / \mathrm{mm}$

Table 1. Material properties for Aluminium

\begin{tabular}{cc}
\hline Young Modulus & $\mathrm{E}=67000 \mathrm{~N} / \mathrm{mm}^{2}$ \\
Poisson Ratio & $v=0.33$ \\
Density & $\rho=2700 \mathrm{~kg} / \mathrm{m}^{3}$ \\
Yield Stress & $\sigma_{\mathrm{v}}=150 \mathrm{~N} / \mathrm{mm}^{2}$ \\
Hardening parameter & $h=44.7 \mathrm{~N} / \mathrm{mm}^{2}$ \\
\hline
\end{tabular}

Table 2. Numerical parameters for time marching algorithms

Implicit scheme : Chung Hulbert parameters $\quad \gamma=0.5 ; \beta=0.25 ; \alpha_{\mathrm{F}}=0.01 \alpha_{\mathrm{M}}=-0.97$

The tube has been modelled using 300 quadrilateral elements $(3 \times 100)$ with 4 Gauss points and constant pressure to avoid locking. It is driven against a $3.97 \mathrm{~mm}$ radius die made of mild steel at a velocity of $40 \mathrm{~m} / \mathrm{s}(144 \mathrm{Km} / \mathrm{h})$. Thus a $50 \mathrm{~mm}$ prescribed vertical displacement over a time period of 0.00125 seconds is imposed on the upper nodes of the tube.

The history of the deformation (frictionless case) is given in figure 2 and a comparison of the final configurations for the different friction coefficients $(\mu=0.0 ; 0.15 \& 0.30)$ is given in figure 3 . In figure 4 are displayed the time/load curves obtained for the three coefficients of friction. 


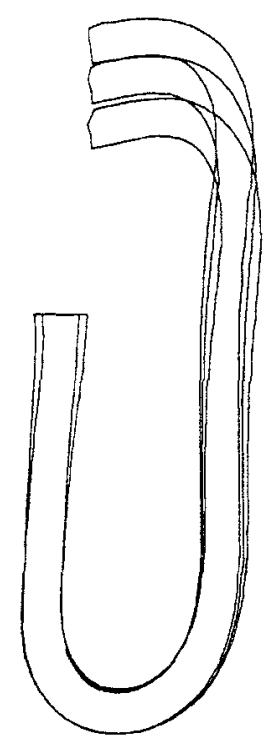

Figure 3: Comparison of the final configurations as a function of friction coefficient.

( $\mu=0.0$ : upper configuration; $\mu=0.15$ : intermediate configuration ; $\mu=0.3$ : lower configuration)

The maximal gap (penetration if frictionless, combination of tangential and normal gaps if not) encountered during the iterative resolution is plot versus the CPU-cost for the Penalty method with the normal penalty parameter $\varepsilon_{N}$ varying from $10^{7}$ to $10^{10}$, and for the Augmented Lagrangian method working with different fixed normal penalty parameter $\left(\varepsilon_{N}=10^{i}\right.$ for AugLag_i $)$ and for several desired precision. This precision is actually given by the corresponding "gap max" values in figures 5 to 7 . The Augmented Lagrangian curves fit the Penalty curve when the desired precision is lower or equal to the precision given by the Penalty method for the same Penalty parameters, i.e. the Augmented Lagrangian method simply reduces to the Penalty method without additional cost and with the same minimal precision. The results are shown for the three cases of friction $(\mu=0.0 ; 0.15 \& 0.30)$ in figures 5, 6 and 7 .

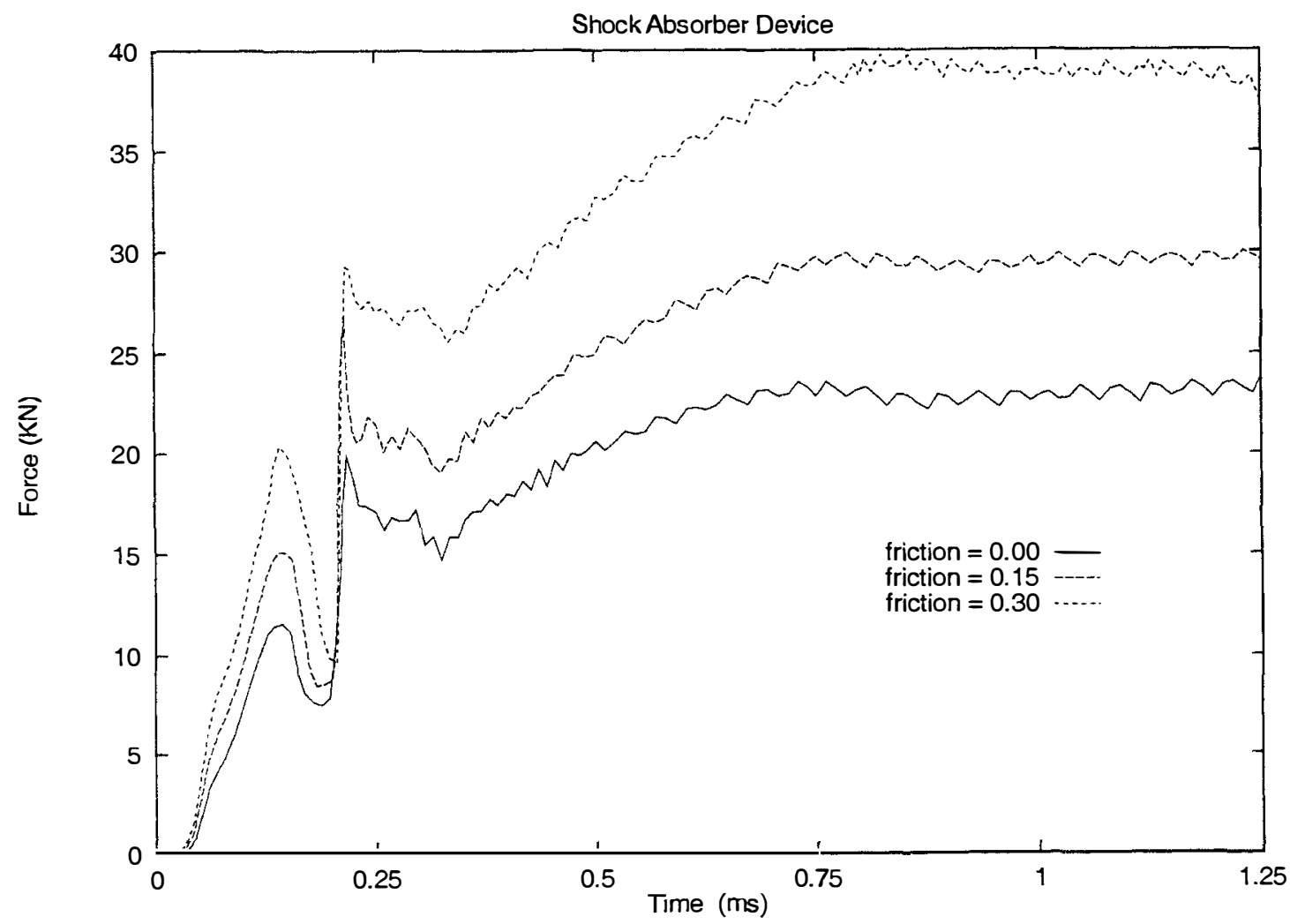

Figure 4: Applied load as a function of time for different friction coefficients 
As it can be obviously seen on figures 5 to 7, with or without friction, the Augmented Lagrangian method allows to reach better accuracy (the Penalty method could not run with higher penalty than approximately $10^{10}$ giving a maximal gap greater than $10^{-7} \mathrm{~mm}$ ) and, moreover, generally produces a given precision at lower cost. In fact, almost all the Augmented Lagrangian curves are on the left side of the Penalty curve, except the lower precision part of the first Augmented Lagrangian curve (AugLag_7) working with the lowest penalty parameter $\left(\varepsilon_{N}=10^{7}\right)$. It's then cheaper to increase the precision by augmenting the Penalty parameters then using the Augmented Lagrangian for these low values of the Penalty parameters. It's only when the influence of these parameters on the conditioning of the tangent stiffness matrix arise then it's worth using the Augmented Lagrangian, otherwise the increase in the number of iterations needed by each augmentations of the Augmented Lagrangian is more expensive then the increase due to the light increase of the ill-conditioning of the stiffness matrix.

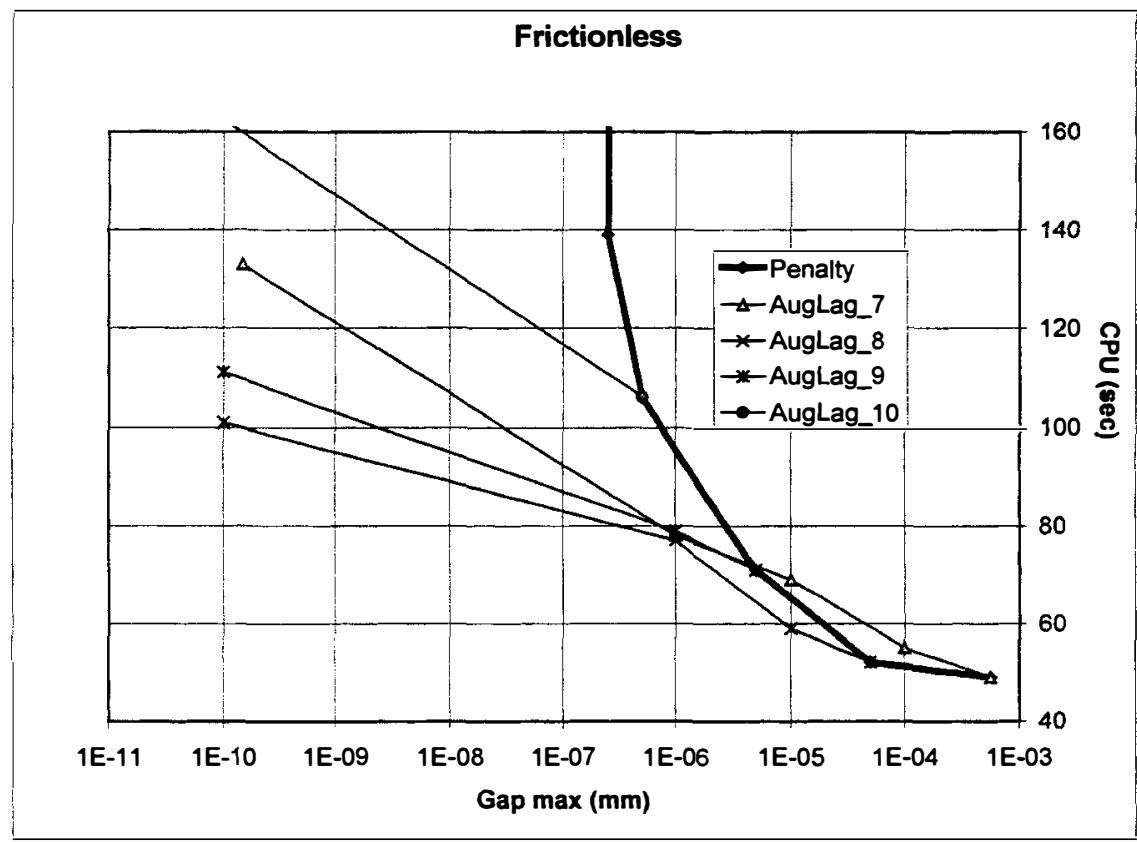

Figure 5: CPU-cost vs precision without friction

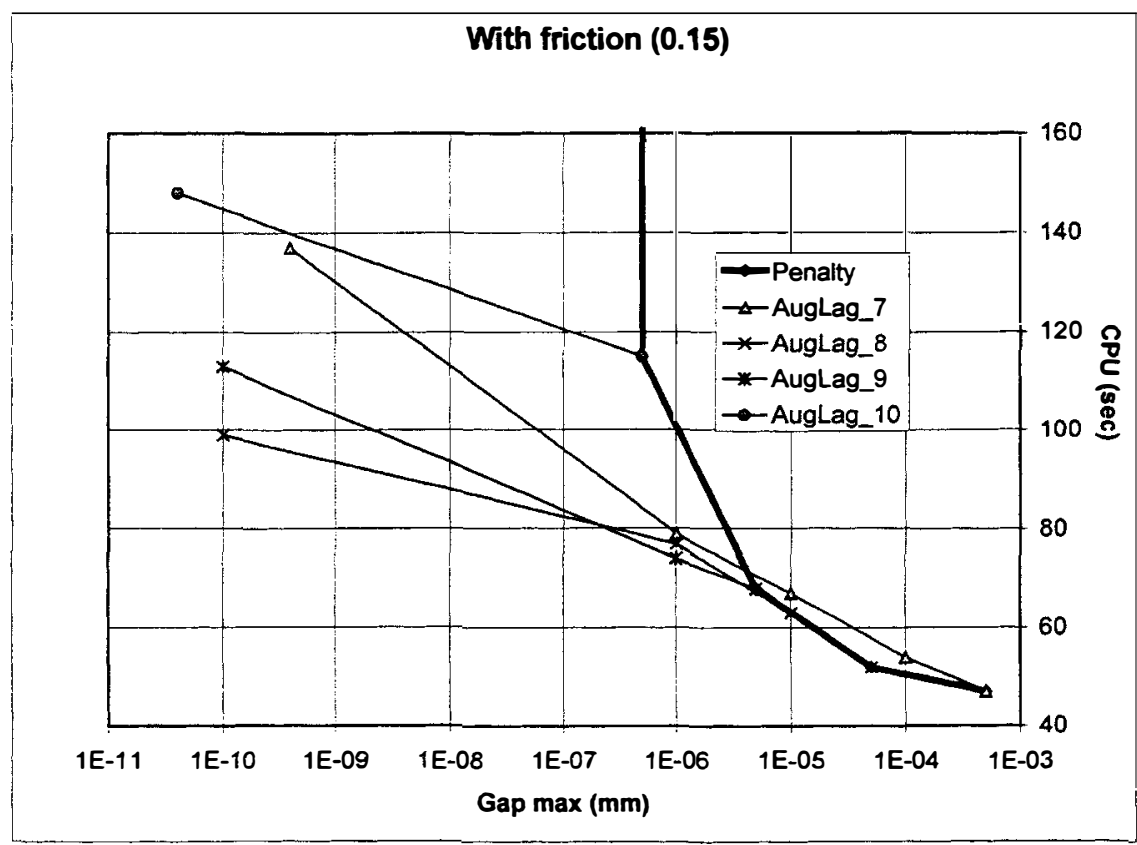

Figure 6: CPU-cost vs precision with friction $(\mu=0.15)$ 


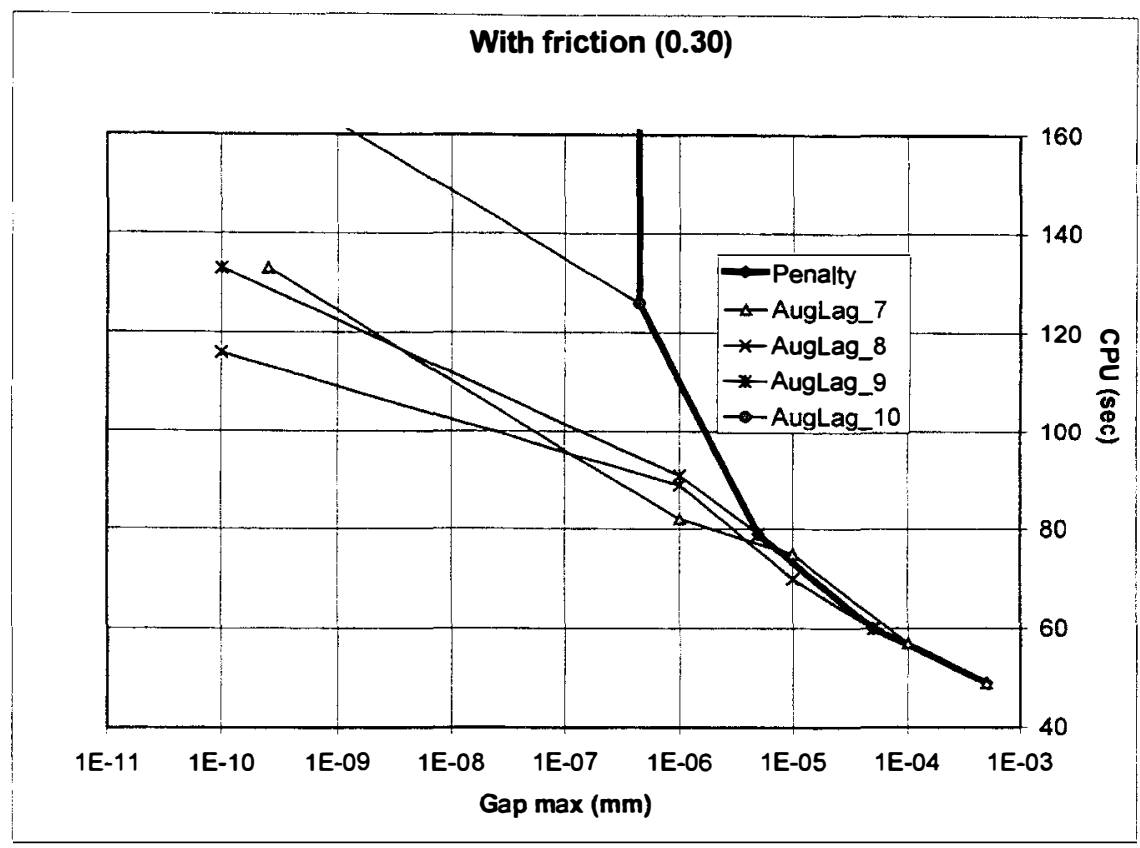

Figure 7: CPU-cost vs precision with friction $(\mu=0.30)$

In table 3 are written the time steps and the iterations needed by the Penalty method and the Augmented Lagrangian at different precision levels for a same penalty parameter $10^{8}$ for the frictional case $(\mu=0.15)$. The number of time steps remains quite the same while the additional number of iterations is induced by the augmentations.

Table 3. Comparison between Penalty and Augmented Lagrangian:

\begin{tabular}{lcccccc}
\hline Method & $\begin{array}{c}\text { Normal penalty } \\
(\mathrm{N} / \mathrm{mm})\end{array}$ & $\begin{array}{c}\text { Precision } \\
(\mathrm{mm})\end{array}$ & Time steps & Iterations & $\begin{array}{c}\text { Mean number of } \\
\text { augmentations }\end{array}$ & $\begin{array}{c}\text { CPU } \\
(\mathrm{sec})\end{array}$ \\
\hline Penalty & $1 . \mathrm{E} 8$ & $5, \mathrm{E}-05$ & 611 & 1873 & $/$ & 60 \\
\hline AugLag & $1 . \mathrm{E} 8$ & $1, \mathrm{E}-05$ & 628 & 2192 & 0.2 & 70 \\
AugLag & $1 . \mathrm{E} 8$ & $1, \mathrm{E}-06$ & 623 & 2752 & 0.9 & 89 \\
AugLag & $1 . \mathrm{E} 8$ & $1, \mathrm{E}-10$ & 616 & 3553 & 2.2 & 116 \\
\hline
\end{tabular}

\section{Dynamic buckling of a cylinder}

This second example deals with the buckling of a thin steel cylinder by axial compression into a rigid matrix. This problem has been first mentioned by Tod A. Laursen [6] who compares the results with and without friction in a quasi-static situation.

In this axisymmetric problem, a steel cylinder is forced downwards (via displacement control of its top surface) into a rigid fixture. The steel is modelled by an elastoplastic law with linear isotropic hardening and has the material properties shown in table 4. Spatial discretization of the cylinder is achieved using 177 bilinear four-nodes elements $(3 \times 59)$ with constant pressure. As the calculation proceeds, the cylinder initially moves down into the die, but when a critical axial load is reached, it begins a series of buckles, as shown in Figure 8. Importantly, this buckling occurs without any initial geometric imperfections. The contact is considered frictionless and is solved using the Penalty method. Information about the initial geometry is given in table 5.

The cylinder is crashed down of $110 \mathrm{~mm}$ in $11 \mathrm{~ms}(10 \mathrm{~m} / \mathrm{s}$ at constant velocity). The problem has been solved using a large set of Penalty parameters ranging from $10^{8} \mathrm{~N} / \mathrm{mm}$ (which gives a maximum penetration encountered lower than $1 \mu \mathrm{m}$ ) to $10^{12} \mathrm{~N} / \mathrm{mm}$ (maximal value before failure of the process). 
Table 4. Material properties for Steel

\begin{tabular}{cc}
\hline Young Modulus & $\mathrm{E}=210000 \mathrm{~N} / \mathrm{mm}^{2}$ \\
Poisson Ratio & $v=0.3$ \\
Density & $\rho=7850 \mathrm{~kg} / \mathrm{m}^{3}$ \\
Yield Stress & $\sigma_{\mathrm{v}}=700 \mathrm{~N} / \mathrm{mm}^{2}$ \\
Hardening parameter & $h=808 \mathrm{~N} / \mathrm{mm}^{2}$ \\
\hline
\end{tabular}

Table 5. Geometrical properties of the cylinder

\begin{tabular}{cc}
\hline Internal Diameter & $D_{\text {int }}=27.00 \mathrm{~mm}$ \\
External Diameter & $D_{\text {ext }}=31.75 \mathrm{~mm}$ \\
Thickness & $e=4.75 \mathrm{~mm}$ \\
Height & $h=180.00 \mathrm{~mm}$ \\
\hline
\end{tabular}

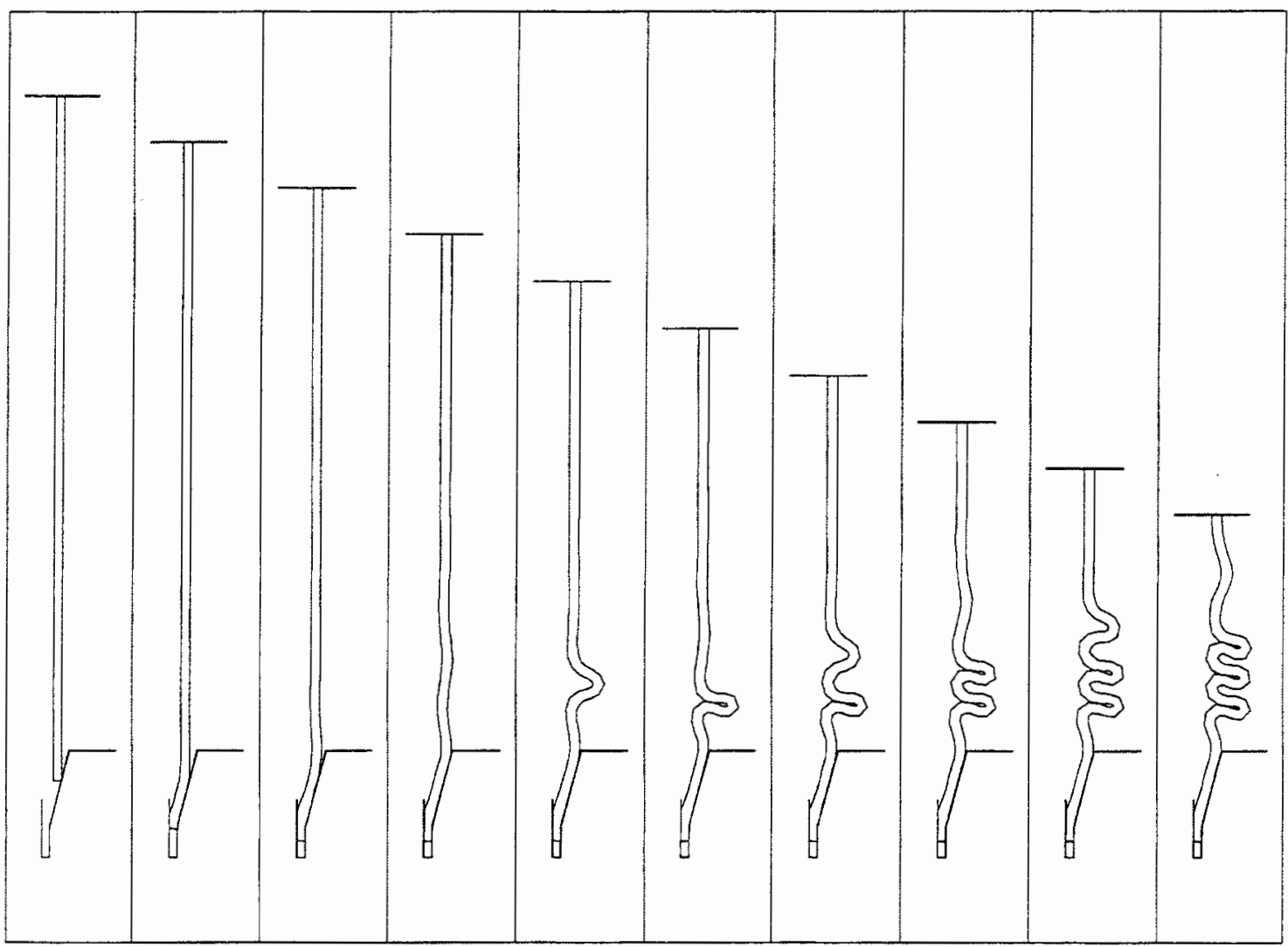

Figure 8: Buckling of a cylinder, configurations from 0 to 11 milliseconds

The maximal gap encountered during the iterative resolution is plotted versus the CPU-cost for the Penalty method with the normal penalty parameter $\varepsilon_{N}$ varying from $10^{8}$ to $10^{13}$, and for the Augmented Lagrangian method working with different fixed normal penalty parameter $\left(\varepsilon_{N}=10^{i}\right.$ for AugLag $\left.i\right)$ and for several desired precision. This precision is actually given by the corresponding "gap max" values in figures 9 and 10. The Augmented Lagrangian curves join again the Penalty curve for a desired precision already reached by the Penalty method. The results are computed with and without friction $(\mu=0.0 ; 0.15)$ in figures 9 and 10 .

As it can be seen again on figures 9 and 10, with or without friction, the Augmented Lagrangian method allows to reach better accuracy (the Penalty method could not run with higher penalty than approximately $10^{12}$ giving a maximal gap greater than $10^{-8} \mathrm{~mm}$ ) and, moreover, generally produces a given precision at lower cost. In fact, all the Augmented Lagrangian curves are on the left side of the Penalty curve. If lower penalty parameters had been used, we would have seen again the low 
precision part of these Augmented Lagrangian curves on the right side of the Penalty curve, for the same reason than previously.

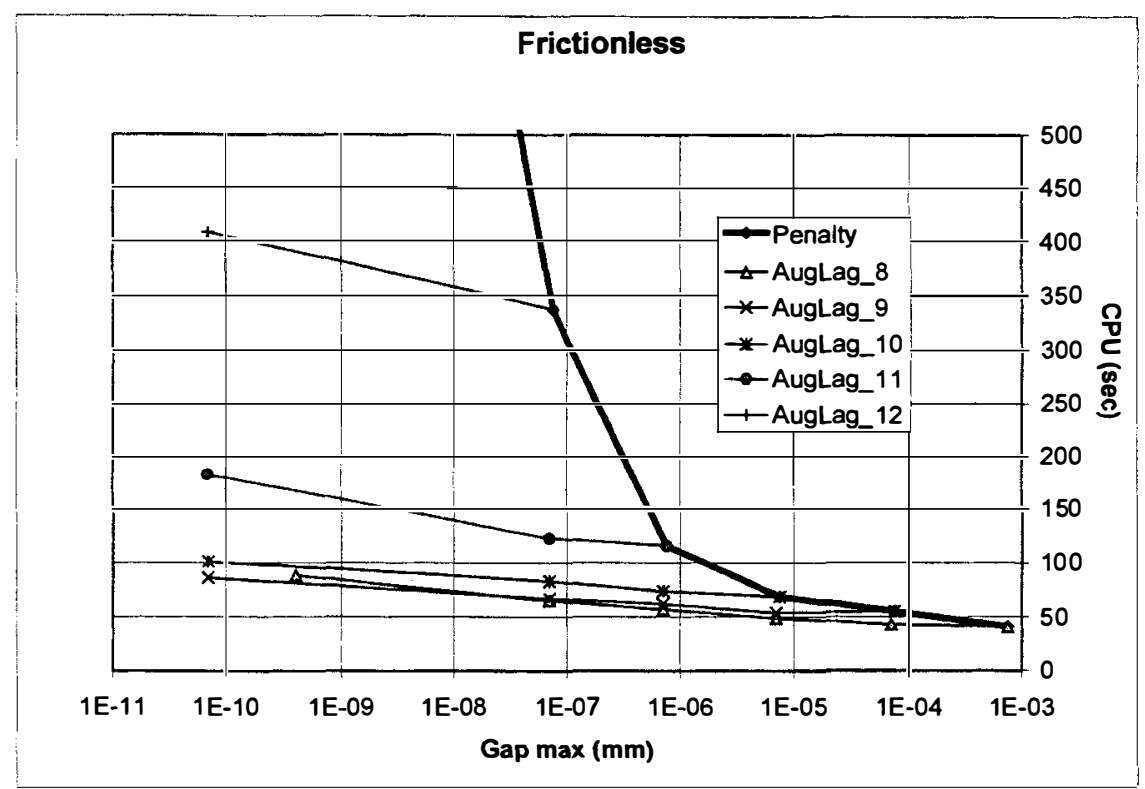

Figure 9: CPU-cost vs precision without friction

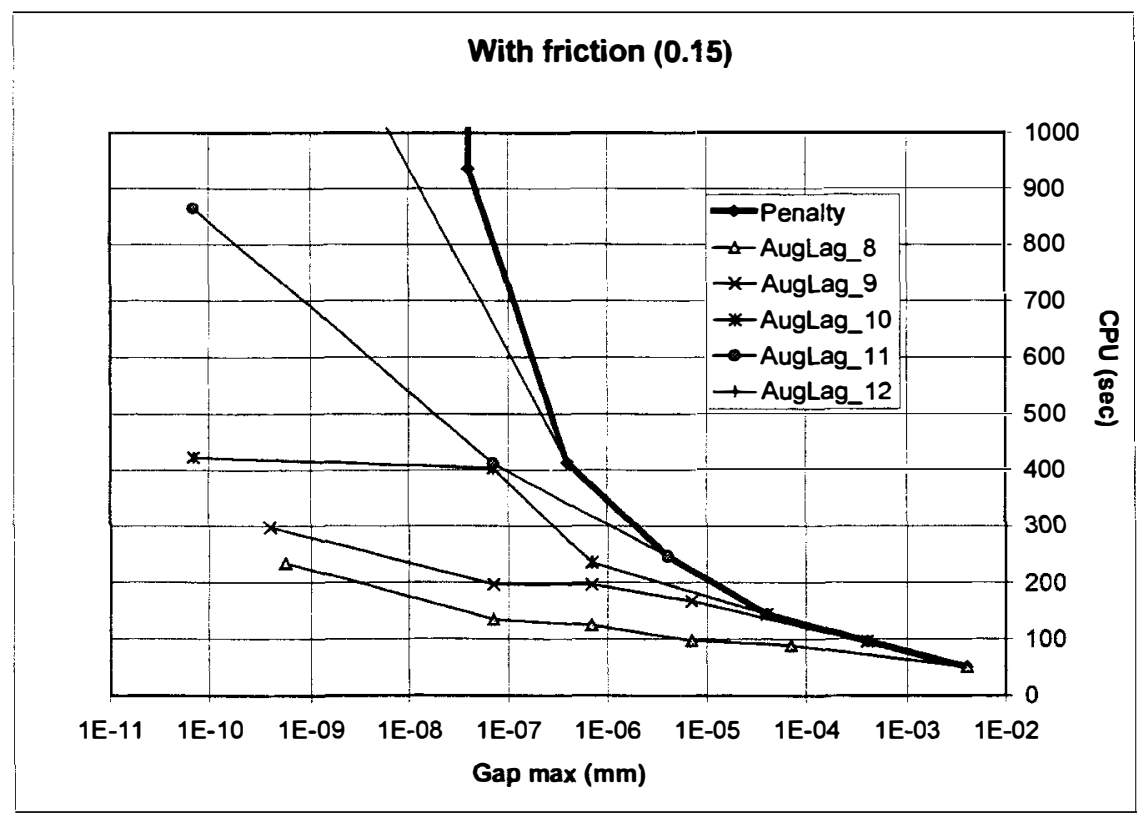

Figure 10: CPU-cost vs precision with friction $(\mu=0.15)$

In table 6 are written the time steps and the iterations needed by the Penalty method and the Augmented Lagrangian at different precision levels for a same penalty parameter $10^{8}$ for the frictional case. The number of time steps remains quite the same while the additional number of iterations is induced by the augmentations. 
Table 6. Comparison between Penalty and Augmented Lagrangian:

\begin{tabular}{ccccccc}
\hline Method & $\begin{array}{c}\text { Normal penalty } \\
(\mathrm{N} / \mathrm{mm})\end{array}$ & $\begin{array}{c}\text { Precision } \\
(\mathrm{mm})\end{array}$ & Time steps & Iterations & $\begin{array}{c}\text { Mean number of } \\
\text { augmentations }\end{array}$ & $\begin{array}{c}\text { CPU } \\
(\mathrm{sec})\end{array}$ \\
\hline Penalty & $1, \mathrm{E}+08$ & $7, \mathrm{E}-04$ & 616 & 1848 & $/$ & 41 \\
\hline AugLag & $1, \mathrm{E}+08$ & $7, \mathrm{E}-05$ & 610 & 1929 & 0,1 & 43 \\
AugLag & $1, \mathrm{E}+08$ & $7, \mathrm{E}-06$ & 582 & 2125 & 0,6 & 49 \\
AugLag & $1, \mathrm{E}+08$ & $7, \mathrm{E}-07$ & 611 & 2469 & 1,0 & 57 \\
AugLag & $1, \mathrm{E}+08$ & $7, \mathrm{E}-08$ & 606 & 2775 & 1,6 & 65 \\
AugLag & $1, \mathrm{E}+08$ & $7, \mathrm{E}-11$ & 606 & 3699 & 3,1 & 88 \\
\hline
\end{tabular}

\section{CONCLUSIONS}

In this paper, it has been shown on two examples that using the Augmented Lagrangian to solve the constrained frictional contact problem can produce significant savings of time and allows to reach a higher precision than the classical Penalty method. An efficient automation technique based on multiple criteria is proposed that produces a desired quality solution at optimal cost. Limits of the method are shown: one must not use too low penalty parameters inducing low efficiency of the augmentation and more additional CPU-cost than simply increasing the penalty parameters in the Penalty method.

\section{REFERENCES}

[1] J-P. Ponthot and M. Hogge. On relative merits of implicit / explicit algorithms for transient problems in metal forming simulation. In International Conference on Numerical Methods for Metal Forming in Industry, Vol. 2, pp 128-148, BadenBaden, Germany, Sept. 1994.

[2] M. Hogge and J-P. Ponthot. Efficient implicit schemes for transient problems in metal forming simulation. In NUPHYMAT'96, Numerical and Physical Study of Material Forming Processes, CEMEF - Ecole Nationale supérieure des Mines de Paris Sophia-Antipolis, France, June 5-7, 1996.

[3] J-P. Ponthot. Mode d'emploi pour la version pilote de METAFOR, module de calcul en grandes déformations. Technical Report TF-24 (in French), Université de Liège, LTAS, 1992.

[4] Beltrán F. and Goicolea J. Large strain plastic collapse: a comparison of explicit and implicit solutions. In Owen R., Hinton H. and Onate E., editor, International Conference on Computational Plasticity, COMPLAS II, pp 1125-1136, Barcelona, Spain, 1989.

[5] C. García Garino. Un modelo numerico para el analysis de solidos elastoplasticos sometidos a grandes deformaciones. PhD thesis (in Spanish), E.T.S. Ingenieros de Caminos, UPC, Barcelona, Spain, 1993.

[6] Tod-A. Laursen. Formulation and Treatment of Frictional Contact Problems using Finite Elements. PhD thesis, Department of Mechanical Engineering, Stanford University, 1992.

[7] Laursen T.A. and Oancea V.G. Automation and Assessment of Augmented Lagrangian Algorithms for Frictional Contact Problems. In Transaction of ASME, Vol. 61, pp 956-963, December 1994.

[8] Landers J.A. and Taylor R.L. An Augmented Lagrangian Formulation for the Finite Element Solution of Contact Problems. NCEL Contract Report, Naval Civil Engineering Laboratory, Port Hueneme, CA, 1986.

[9] Simo J.C. and Laursen T.A. An Augmented Lagrangian Treatment of Contact Problems involving Friction. Computers and Structures, Vol 42, pp97-116, 1992.

[10] Wriggers P. and Zavarise G. On the Application of Augmented Lagrangian Techniques for Nonlinear Constitutive Laws in Contact Interfaces. Communications in Applied Numerical Methods, submitted for publication, 1993.

[11] Heegaard J.H. and Curnier A. An Augmented Lagrangian Method for Discrete Large-Slip Contact Problems. International Joumal for Numerical Methods in Engineering, Vol 36, pp 569-593, 1993.

[12] Bertsekas D. Constrained Optimization and Lagrange Multiplier Methods. Athena scientific, 1996. 